\title{
Treatment with high doses of pegvisomant in 56 patients with acromegaly: experience from ACROSTUDY
}

\section{Aart Jan van der Lely', Peter Jönsson², Patrick Wilton³, Ann-Charlotte Åkerblad², José Cara ${ }^{4}$ and Ezio Ghigo ${ }^{5}$}

'Department of Medicine, Erasmus University MC, Rotterdam, The Netherlands, ${ }^{2}$ Pfizer Endocrine Care, Pfizer Health AB, Sollentuna, Sweden, ${ }^{3}$ Pfizer Endocrine Care, Stockholm, Sweden, ${ }^{4}$ Endocrine Care, Pfizer Inc., New York, New York, USA, and ${ }^{5}$ University of Turin, Turin, Italy
Correspondence should be addressed to A J van der Lely Email a.vanderlelij@erasmusmc.nl

\begin{abstract}
Objective: To investigate the characteristics of patients who need more or less pegvisomant (PEGV) to normalize serum IGF-I.

Design: ACROSTUDY is a global noninterventional safety surveillance study of long-term treatment outcomes in patients treated with PEGV. As of June, 2014, ACROSTUDY included data on 2016 patients. All patients treated for at least 6 weeks at a dose above $30 \mathrm{mg} /$ day and who had two consecutive normal serum IGF-I values were included in the 'high'-dose group ( $\mathrm{H} ; n=56$; mean daily dose $44 \pm 12.5$; median dose 40, 35-60 (10-90\%)). Patients with two consecutive normal IGF-I values and who never received a PEGV dose above $10 \mathrm{mg} /$ day were included in the 'low'-dose group ( $L ; n=368$; mean daily dose $7.5 \pm 2.5$; median dose 8.6 , 4.3-10 (10-90\%)).

Results: Patients in the $\mathrm{H}$ group were significantly younger (median 47 vs 52 years) and had a significantly higher BMI (median 31.8 vs $26.5 \mathrm{~kg} / \mathrm{m}^{2}$ ). They had more diabetes ( $55 \%$ vs $21 \%$ ), sleep apnea ( $25 \%$ vs $14 \%$ ) and more hypertension (61\% vs $43 \%)$. The incidence of (serious) adverse events was low and was not different between the groups.

Conclusions: Patients who need more PEGV to normalize IGF-I have more aggressive disease, as they are younger, have higher baseline IGF-I levels, more hypertension, more sleep apnea and diabetes and are more overweight. A better understanding of this dose-efficacy relationship of PEGV might avoid inappropriate dosing and prevent serum IGF-I levels from remaining unnecessarily uncontrolled.
\end{abstract}

European Journal of Endocrinology

(2016) 175, 239-245

\section{Introduction}

Pegvisomant (PEGV) is a growth hormone (GH) receptor antagonist for the treatment of acromegaly. The first reports on safety and efficacy of PEGV were published some 15 years ago $(1,2)$.

ACROSTUDY, established in 2004, is a global noninterventional safety surveillance study of the longterm treatment outcomes in patients treated with PEGV. ACROSTUDY is open to all patients with acromegaly who are treated, or planned to be treated, with pegvisomant according to the routine clinical practice of the physician investigator, and contains a growing volume of data on patients with this rare disease. The objectives of the

www.eje-online.org DOI: $10.1530 / E J E-16-0008$ (c) 2016 European Society of Endocrinology Printed in Great Britain study were to monitor the long-term safety and treatment outcomes of PEGV in patients with acromegaly. An important part of the current data set was provided by the German Pegvisomant Observational Study (GPOS), initiated in January 2004, immediately after pegvisomant received marketing authorization in Germany (3).

In 2012, we reported that ACROSTUDY appeared to be a valuable tool in the assessment of the long-term safety of PEGV for the treatment of acromegaly and that data entered in ACROSTUDY up to 31 December 2009 on 1288 subjects indicated that PEGV is an effective and safe medical treatment in patients with acromegaly,

Published by Bioscientifica Ltd. 
which cannot be controlled by somatostatin analog monotherapy (4).

Available data on the relationship between the dose of PEGV required to normalize serum IGF-I levels in patients with acromegaly have suggested that several clinical, phenotypical, biochemical and other characteristics exist which might more or less 'predict' which patients need higher or lower PEGV dosing to normalize serum IGF-I values. Marazuela and coworkers, for example, reported in 2009 that male gender, previous irradiation and longer treatment duration were associated with a better response to PEGV therapy (5). Also, Parkinson and coworkers reported that women required more PEGV to normalize serum IGF-I values than men, as did patients with a higher body weight (6). Again, they also observed that patients treated with radiotherapy required lower doses of PEGV than patients not radiated (6). In patients cotreated with somatostatin analogs (LA-SRAs), Neggers and coworkers observed in 2007 that baseline serum IGF-I levels were positively correlated with the required dosage of PEGV (7). Finally, Andreassen and coworkers reported that in healthy subjects treated with $15 \mathrm{mg}$ per day of PEGV for 3 weeks, serum PEGV levels were positively correlated with baseline muscle mass and inversely related to baseline fat (8).

Using the largest available database on the use of PEGV, we investigated all available data in ACROSTUDY, as of June 2014, to address factors that influence the necessary dose of PEGV to normalize serum IGF-I concentrations in patients with acromegaly.

\section{Patients and methods}

As described before (4), ACROSTUDY is an ongoing openlabel, global, noninterventional, postmarketing safety surveillance study open to patients with acromegaly who are treated with (or about to begin) PEGV. This report describes data available in ACROSTUDY as of June 2014.

Enrolled patient data were captured by local investigators on electronic case report forms using a web-based data entry tool. Data from routine clinic visits were collected using information available in the clinical records and no additional diagnostic or monitoring procedures were conducted as part of the study.

The protocol recommended the following minimum follow-up evaluations: pituitary imaging at 6 months, 12 months and annually after PEGV treatment start and liver function tests and serum IGF-I levels every 6 months. Only locally measured IGF-I data were reported in relation to local reference values. Both historical and prospective data were collected during the study (4). ACROSTUDY does not use a central laboratorium facility for the IGF-I assessments. Because of this, serum IGF-I concentrations were expressed as IGF-I/upper level of normal (ULN) and a normal serum IGF-I concentration was defined as an IGF-I/ULN below 1.2, as the available IGF-I assays show significant variations (9).

\section{Main criteria for inclusion and exclusion}

As described in earlier reports, ACROSTUDY included data from subjects with acromegaly who were already being treated with PEGV as well as those who were about to start PEGV therapy (4). The most important exclusion criteria were patients participating in any clinical trial of an investigational drug for acromegaly, patients requiring surgical decompression of tumor (such as in contact with the optic chiasm) or patients who should receive nonmedical therapy because of visual field loss, cranial nerve palsies or intracranial hypertension.

The ACROSTUDY data reported here were collected in compliance with, and consistent with, the most recent version of the Declaration of Helsinki. In addition, the study adhered to all applicable local laws and regulatory requirements in the countries involved. Local ethical approval was obtained for all participating centers, and all patients provided written informed consent before any data were captured.

\section{Statistical procedures}

For proportions, the $\chi^{2}$ or Fisher's exact test was used for comparisons. For variables with normal distribution, the Student's $t$-test was used for comparisons; otherwise, the Wilcoxon rank sum test was used. Incidence was calculated using Byar's approximation formula (Poisson assumption). SAS 9.2 was used for all statistical analyses. Values are expressed as mean \pm s.D. unless otherwise noted.

\section{Patients}

All patients treated with pegvisomant for at least 6 weeks at a dose above $30 \mathrm{mg} /$ day and who had two consecutive normal serum IGF-I values were included in the 'high'dose group $(n=56 ; 39 \%$ male; median age $=46.8$ years; median $\mathrm{BMI}=31.8 \mathrm{~kg} / \mathrm{m}^{2}$ ). This dose was chosen, as $30 \mathrm{mg} /$ day is the highest allowed dose according to the label; therefore, this high-dose group represents a subpopulation that really needs a high dose of PEGV. 
The median duration of acromegaly in the $\mathrm{H}$ group was 2.7 years. The $\mathrm{H}$ group underwent surgery in $84 \%$, while $29 \%$ was irradiated in the past. There were 50 patients in the database that used high doses of PEGV, but were not normalized in their IGF-I and, therefore, not included in the analyses. The $\mathrm{H}$ group was compared with patients who had two consecutive normal IGF-I values and who never received pegvisomant treatment with a dose above $10 \mathrm{mg} /$ day. This subpopulation represents the 'Low'-dose group, reflecting a high sensitivity for PEGV $(n=368 ; 46 \%$ male; median age $=51.9$ years; median $\mathrm{BMI}=26.5 \mathrm{~kg} / \mathrm{m}^{2}$ ). The mean duration of acromegaly in the L-group was 5.3 years. The L-group underwent surgery in $77 \%$, while $26 \%$ was irradiated in the past. We decided to compare these extremes to better discriminate which parameters describe best the properties of these two subpopulations. See also Table 1.

Patients were considered to have diabetes mellitus if the treating physicians answered with a 'YES' to the question whether the patient had diabetes on the electronic case report form (e-CRF). In addition, patients were considered to have diabetes if any one of the following three criteria were met: (i) a hemoglobin A1c (HbA1c) test with a value equal to or above $6.5 \%$ before PEGV start; (ii) concomitant antidiabetic medication reported in the e-CRF before PEGV start or (iii) plasma glucose equal to or above $200 \mathrm{mg} / \mathrm{dL}$ (11.1 mmol/L) before PEGV start.

Patients were considered to have hypertension when the treating physicians answered with a 'YES' to the question whether the patient had hypertension on the e-CRF. In addition, patients were considered to have hypertension when any one of the following two criteria were met: (i) the treating physicians provided the reason 'hypertension' in the e-CRF as the indication for the use of concomitant medication; (ii) a systolic blood pressure of more than $130 \mathrm{mmHg}$ or diastolic blood pressure of more than $85 \mathrm{mmHg}$ was recorded in the e-CRF.

\section{Results}

Patients in the high-dose group $(\mathrm{H} ; n=56)$ received a mean daily dose of pegvisomant of $44 \pm 12.5 \mathrm{mg}$; median dose $40 \mathrm{mg}$, range 35-60 mg (10-90\%). Of these, 53 subjects received their dose by daily injections, one by twiceweekly injections and one by four-weekly injections. Patients in the low-dose group ( $\mathrm{L} ; n=368$ ) received a mean daily dose of pegvisomant of $7.5 \pm 2.5 \mathrm{mg}$ (median dose 8.6 , range $4.3-10 \mathrm{mg}$ (10-90\%)). At baseline, the mean daily dose of PEGV (at PEGV start) was 16.6 and $8.8 \mathrm{mg}$ in $\mathrm{H}$ and $\mathrm{L}$ respectively, which was significantly different.

Duration of acromegaly differed significantly between the 56 subjects (39\% males) in $\mathrm{H}$ and the 368 subjects in $\mathrm{L}$ (46\% males) ( $\mathrm{H}$ median and 10-90 percentiles: 2.7 years, $0.3-18.8$ vs L: 5.3 years, $1.0-21.8)(P=0.0028)$. The subjects in $\mathrm{H}$ were significantly younger at PEGV start than in $\mathrm{L}$, as their mean \pm s.D. ages were $43.6 \pm 13.0$ years vs $51.9 \pm 14.6$ years $(P=0.0001)$. The mean PEGV treatment

Table 1 Demographic background and baseline characteristics of 56 acromegaly patients treated for at least 6 weeks at a dose above $30 \mathrm{mg} /$ day and who had two consecutive normal IGF-I values (high-dose group) and 368 patients who had two consecutive normal IGF-I values and who never received treatment with a dose above $10 \mathrm{mg} /$ day (low-dose group). Values are expressed as medians (P10-P90).

\begin{tabular}{l} 
Demographic background and \\
baseline characteristics \\
\hline Age (years) \\
Male $(\%)$ \\
Weight $(\mathrm{kg})$ \\
BMI $\left(\mathrm{kg} / \mathrm{m}^{2}\right)$ \\
Duration acromegaly (years) \\
Surgery $(\%)$ \\
Radiation $(\%)$ \\
DM (\%) \\
Hypertension (\%) \\
Sleep apnea (\%) \\
IGF-I/ULN \\
Elevated IGF-I (\%) \\
\hline
\end{tabular}

\begin{tabular}{|c|c|}
\hline High dose $(n=56)$ & Low dose $(n=368)$ \\
\hline $46.8(26.0-60.2)$ & $51.9(33.2-72.3)$ \\
\hline 39 & 46 \\
\hline $97.8(75.0-125.9)$ & $77(60-100)$ \\
\hline $31.8(26.6-41.7)$ & $26.5(21.8-32.2)$ \\
\hline $2.7(0.3-18.8)$ & $5.3(1.0-21.8)$ \\
\hline 84 & 77 \\
\hline 29 & 26 \\
\hline 55 & 21 \\
\hline 61 & 43 \\
\hline 25 & 14 \\
\hline $2.6(1.2-4.8)$ & $1.3(0.7-2.2)$ \\
\hline 89 & 61 \\
\hline
\end{tabular}

\begin{tabular}{c}
$\begin{array}{c}\text { Significant } \\
\text { difference } \\
\text { (Yes/No) }\end{array}$ \\
\hline Y \\
$N$ \\
Y \\
Y \\
Y \\
$N$ \\
$N$ \\
Y \\
Y \\
$N$ \\
Y \\
Y \\
\hline
\end{tabular}

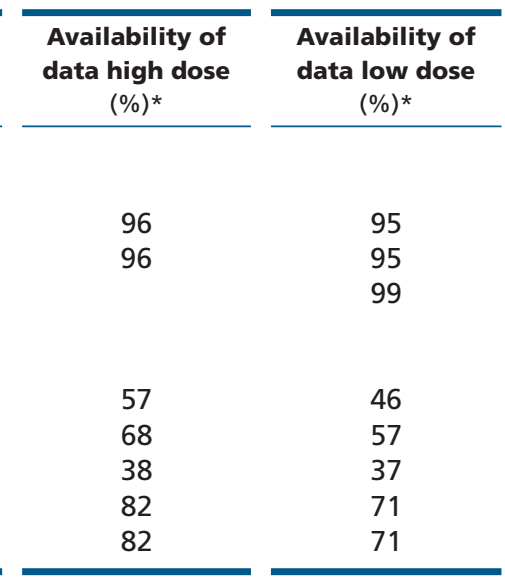

*If blank, data are available for all patients. 
time was $6.9 \pm 2.5$ years (s.D.) for the $H$ group and $4.4 \pm 2.5$ years (S.D.) for the L-group $(P=0.0001)$.

Noteworthy is the fact that of the $56 \mathrm{H}$ subjects, 24 (43\%) received concomitant treatment with long-acting somatostatin analogs (LA-SRAs), a similar rate to that observed in the L subjects (160 (43\%) of 368). In the $\mathrm{H}$ group, 10 of the 56 subjects (18\%) were cotreated with dopamine agonists, against 42 of the 368 subjects (11\%) in the L-group. Also, 1 of the 33 (3\%) females in the $\mathrm{H}$ group received oral estrogens, against 3 of the 196 (2\%) in the L-group. The medical histories of subjects in $\mathrm{H}$ and $L$ revealed small and nonsignificant differences in the percentages of subjects who had undergone neurosurgery ( $84 \%$ in $\mathrm{H}$ vs $77 \%$ in $\mathrm{L}$ ) and radiotherapy (29\% in $\mathrm{H}$ vs $26 \%$ in L).

Subjects in $\mathrm{H}$ had a significant higher body weight vs subjects in L $(98.5 \pm 21.6 \mathrm{~kg}$ vs $78.4 \pm 16.0 \mathrm{~kg} ; P=0.0001)$. This resulted in equally significant differences in body mass index (BMI), which was $33.7 \pm 6.7 \mathrm{~kg} / \mathrm{m}^{2}$ in $\mathrm{H}$ and $26.9 \pm 4.3 \mathrm{~kg} / \mathrm{m}^{2}$ in $\mathrm{L}(P=0.0001)$. Another significant difference between subjects in $\mathrm{H}$ and $\mathrm{L}$ was the ratio between IGF-I/ULN before start of PEGV treatment: In subjects in $H$, the ratio was $2.6 \pm 1.3$, and in subjects in $\mathrm{L}$, the ratio was $1.3 \pm 0.7(P=0.0001)$. These ratios were available for $82 \%$ of patients in $\mathrm{H}$ and $71 \%$ in $\mathrm{L}$.

The presence of comorbidities was examined in both patient groups. The coexistence of diabetes was confirmed by the treating physician in $55 \%$ of the $57 \%$ of subjects in $\mathrm{H}$ for whom data on the presence of diabetes were recorded. For the $46 \%$ of subjects in $\mathrm{L}$ for whom data were recorded, diabetes was confirmed in $21 \%(P<0.0001)$. In regards to hypertension, $61 \%$ of the $68 \%$ of subjects in
$\mathrm{H}$ for whom data were recorded had hypertension. For the $57 \%$ of subjects in L for whom data were recorded, hypertension was present in $43 \%(P=0.0369)$. Finally, for sleep apnea, these figures were $25 \%$ of $38 \%$ of subjects with data available in $\mathrm{H}$ and $14 \%$ of $37 \%$ of subjects with data available in $\mathrm{L}(P=0.0647)$.

In $\mathrm{H}, 33$ serious adverse events (SAEs) were recorded in 16 subjects during 389 patient treatment years (PTYs) of follow-up, which results in an incidence of 70/1000 PTYs (CI: 54-80). Four patients were withdrawn because of AEs, corresponding to an incidence of 10.3/1000 PTYs. Of these four, three were reported as recovered and one was reported as not recovered.

In $\mathrm{L}, 113$ SAEs were reported in 67 patients during 1612 PTYs of follow-up, resulting in an incidence of 84/1000 PTYs (CI: 48-104). Thirty-seven patients were withdrawn because of AEs (23.0/1000 PTY). Of these 37,13 were reported as recovered, 1 as recovered with sequelae, 10 as not recovered, 9 as death, 2 as recovering, 1 as unknown and information not available for 1 patient. In Table 2, the occurrence of the most common adverse events (AEs) is provided, which shows that the incidence of AEs is low in both groups. When evaluated by sex, the number of women in the $\mathrm{H}$ group with any AEs reported was higher than men, as 'only' 11 of the 22 men reported any AE compared with 29 of the 34 women; $P<0.005$. All AE occurred on doses $>30 \mathrm{mg} /$ day, except a few. Only 1 subject with transaminase elevations used less than $30 \mathrm{mg}$ daily when the AE was recorded; one subject with arthralgia at $20 \mathrm{mg}$, one with hypertension at $20 \mathrm{mg}$ and one recurrence of pituitary adenoma at $20 \mathrm{mg}$. The analyses contain all 15 patients with transaminase elevations,

Table 2 The occurrence of the most common adverse events in 56 acromegaly patients treated for at least 6 weeks at a dose above $30 \mathrm{mg} / \mathrm{day}$ and who had two consecutive normal IGF-I values (high-dose group) and 368 patients who had two consecutive normal IGF-I values and who never received treatment with a dose above $10 \mathrm{mg} / \mathrm{day}$ (low-dose group).

\begin{tabular}{|c|c|c|c|c|}
\hline & \multicolumn{2}{|c|}{ High-dose group } & \multicolumn{2}{|c|}{ Low-dose group } \\
\hline & No of AEs & Incidence/1000 & No of AEs & Incidence/1000 \\
\hline Increased IGF-I & 15 & 38.5 & 20 & 12.4 \\
\hline Vitamin D deficiency & 6 & 15.4 & 4 & 2.5 \\
\hline Elevated liver enzymes & 5 & 12.9 & 12 & 7.4 \\
\hline Hypertension & 5 & 12.9 & 9 & 5.6 \\
\hline Pituitary recurrence & 4 & 10.3 & 15 & 9.3 \\
\hline Arthralgia & 4 & 10.3 & 14 & 8.7 \\
\hline Diabetes & 3 & 7.7 & 4 & 2.5 \\
\hline Cholelithiasis & 3 & 7.7 & 3 & 1.9 \\
\hline Osteoarthritis & 2 & 5.1 & 7 & 4.3 \\
\hline Sleep apnea & 2 & 5.1 & 1 & 0.6 \\
\hline Injection site reactions & 1 & 2.6 & 13 & 8.1 \\
\hline Arthritis & 1 & 2.6 & 2 & 1.2 \\
\hline Cholecystitis & 0 & 0 & 1 & 0.6 \\
\hline
\end{tabular}

www.eje-online.org 
three in high dose and 12 in low dose (reference group). Surgery for gallstones was reported in 2 of the 3 in the HD and 3 of the 12 in the LD groups respectively. One in high reported as 'hepatic steatosis' and one in the low group as 'hepatic cyst'. Somatostatin analogs, which were linked to higher incidence of increases transaminases, were used at the time of the transaminases $\mathrm{AE}$ by 1 of 3 in the $\mathrm{HD}$ and 6 of the 12 in the LD groups respectively.

\section{Discussion}

In this subanalysis of data in ACROSTUDY, we observed that patients who needed high doses of PEGV to normalize serum IGF-I levels appeared to have a more severe form of acromegaly than patients who required low doses of the drug. Before start of PEGV treatment, they were younger and they had a higher incidence of hypertension, sleep apnea and diabetes. Using the large set of data from ACROSTUDY, we were able to collect data in 56 patients who all used PEGV dosages of 35-60 mg/day, exceeding the highest allowed dose of $30 \mathrm{mg}$ daily that is mentioned in the label.

In our comprehensive review of the long-term safety of PEGV in 1288 subjects enrolled in ACROSTUDY (4), we reported that after 5 years of PEGV treatment, 63.2\% of subjects had normal serum IGF-I levels, while serum IGF-I levels remained elevated in 34\%. The proportion of patients with normal serum IGF-I levels remained stable over time. The mean dosages after 5 years, expressed as $\mathrm{mg} /$ day, were 18 for the well-controlled group and 20 for the uncontrolled group (4). These data, combined with the data in the present analysis, suggest that PEGV is effective in most patients, provided that the medication is titrated appropriate and the appropriate dose is used.

It is noteworthy that we performed a specific analysis of a predefined cohort of subjects who needed a high dose to evaluate their characteristics relative to individuals who need a 'low' dose of pegvisomant to control IGF-I values. When we would have included all subjects, knowing the fact that around 1:3 subjects are not controlled, probably due to 'underdosing' by the treating physicians, we could not have distinguished these characteristics, lacking the information on who needs what particular dose.

Differences in therapeutic response to PEGV treatment of acromegaly have been noted by other authors. Male gender, previous irradiation and treatment duration were previously reported to be associated with a better response to PEGV therapy (5). In our present analysis, we could not confirm these findings, as gender, history of radiotherapy and treatment duration were not different between wellcontrolled subjects in $\mathrm{H}$ and $\mathrm{L}$.

The analysis is confined to patients in whom IGF-I normalized on PEGV therapy which introduces the potential for bias, e.g. the younger age of the highdose cohort may reflect a greater desire of treating physicians to achieve a normal IGF-I in a younger person or alternatively a willingness to spend more on the treatment of a younger patient. Therefore, this analysis was specifically done in patients who were in control (this was part of the specific patient selection criteria), as we could not exclude the possibility that patients who were not in control were not yet titrated to an appropriate pegvisomant dose.

The observed higher incidence of diabetes in the $\mathrm{H}$ group is noteworthy. Although we do not have information on the proportion of type 1 vs type 2 diabetes subjects, we assume that most are patients with type 2 diabetes who are severely insulin resistant due to the high $\mathrm{GH}$ action. In the HD group, one subject had diabetes, but did not use SSAs. In the LD group, six had diabetes, of which only one subjects used SSA analog treatment.

Droste and coworkers already reported interdependences between the acromegaly, the glucose metabolism of predisposed patients and their treatment with pegvisomant. They observed that patients with concomitant diabetes mellitus, in particular those receiving insulin therapy, require higher pegvisomant doses to normalize IGF-I (10).

These observations that subjects with type 2 diabetes need more PEGV fits into the concept that the high portal insulin levels likely to be present in these insulin resistant type 2 diabetes subjects make the liver more GH sensitive (11). This phenomenon was reported by Leung and coworkers in 2000, suggesting that insulin regulates hepatic GH receptor biosynthesis and surface translocation in a reciprocal manner, with surface receptor availability of the net result of these divergent effects. The divergent actions of insulin appear to be mediated by the mitogenactivated protein kinase and phosphatidylinositol 3-kinase pathways respectively (11).

It is noteworthy that in the high-dose cohort that contains younger patients with more aggressive disease, $16 \%$ had never had pituitary surgery. However, due to the observational nature of the database, there was no way to influence the actual treatment choices by physicians for their patients. What might be the case, however, is that for example some patients have tumors that have infiltrated the cavernal sinus, which makes surgical intervention by definition noncurative, but we do not have data on this. 
Our observation of higher disease activity in subjects in $\mathrm{H}$, who showed significantly more frequent hypertension, diabetes and sleep apnea, together with higher baseline serum IGF-I concentrations, was also reported by Parkinson and coworkers (6). They observed a significant correlation between baseline GH, serum IGF-I values, body weight and the dose of pegvisomant required to normalize serum IGF-I concentrations (6). Their data were collected from 69 men and 49 women participating in multicenter, open-label trials of pegvisomant who were retrospectively evaluated using multiple regression techniques (6).

We observed identical proportions of subjects concomitantly treated with LA-SRAs in both the $\mathrm{H}$ and $\mathrm{L}$ groups. By defining $\mathrm{H}$ and $\mathrm{L}$ using PEGV dosages as the key parameter, we are not able to identify whether concomitant treatment with LA-SRAs had any effect on the necessary dose of PEGV.

Overall, our data indicate that young patients with acromegaly who have high serum IGF-I levels, who suffer more frequently from hypertension and sleep apnea, and who also have diabetes are very likely subjects who need more PEGV than subjects who do not suffer from these comorbidities. Clinicians should be aware of the implications of these findings, as they should titrate the dose of PEGV accordingly when the serum IGF-I levels remain elevated.

As importantly, there were no unexpected safety issues in subjects in either the $\mathrm{H}$ or L groups, and the incidence and the type of AEs and SAEs were not different between groups. Interestingly, the number of women with any AEs reported in $\mathrm{H}$ was higher than the number of men with reported AEs in $\mathrm{H}$. This sex-dependent difference has not been reported previously during PEGV treatment and may be related to the small number of subjects in the $\mathrm{H}$ group.

A potential question could be whether or not the analyses include an intrinsic bias in that patients on high dose will have been dose titrated through lowdose therapy and, therefore, it represents a selected cohort that did not experience problems while on low dose. However, all subjects analyzed were selected by dose (high vs low), provided that their IGF-I had been normalized. Therefore, all of them were similar in IGF-I levels and were, therefore, all biochemically controlled in disease activity. The differences in patient's characteristics between the two groups now reflect the likelihood of needing high vs low dose. Therefore, we do not suspect a significant intrinsic bias.

In conclusion, analyses of data from ACROSTUDY, comparing acromegaly patients that need high dosages of
PEGV vs ones that need low dosages of PEGV to normalize their pathognomonic increased serum IGF-I levels, indicate that high-dose PEGV treatment is necessary in patients with higher disease activity. This higher disease activity was defined as having more hypertension, more diabetes, more sleep apnea and higher pre-PEGV treatment serum IGF-I levels. These patients are also significantly younger. These data might help treating physicians modify their dose regimens of PEGV when treating patients with acromegaly.

Declaration of interest

$A J v d L$ and $E$ G received honoraria from Pfizer as members of the ACROSTUDY Strategic Advisory Board. P W is an employee of Vision IT, who was a paid contractor to Pfizer Inc. in the development of this manuscript. A-C A was a full-time employee at Pfizer Health $A B$ when the work was done. P J and J C are full-time employees of Pfizer. The authors were not paid for their contribution to this manuscript.

Funding

ACROSTUDY $^{\mathrm{TM}}$ is owned and financed by Pfizer Inc.

\section{Acknowledgements}

The authors wish to thank all of the investigators, subinvestigators, nurses and study coordinators involved in ACROSTUDY and the patients who allowed their data to be captured. ACROSTUDY is sponsored by Pfizer Inc.

\section{References}

1 Trainer P, Drake W, Katznelson L, Freda P, Herman-Bonert V, Lely A, Dimaraki E, Stewart P, Friend K \& Vance M. Treatment of acromegaly with the growth hormone-receptor antagonist pegvisomant. New England Journal of Medicine 2000342 1171-1177. (doi:10.1056/NEJM200004203421604)

2 van der Lely AJ, Hutson RK, Trainer PJ, Besser GM, Barkan AL, Katznelson L, Klibanski A, Herman-Bonert V, Melmed S, Vance ML et al. Long-term treatment of acromegaly with pegvisomant, a growth hormone receptor antagonist. Lancet 2001358 1754-1759. (doi:10.1016/s0140-6736(01)06844-1)

3 Trainer PJ. ACROSTUDY: the first 5 years. European Journal of Endocrinology 2009161 (Supplement 1) S19-S24. (doi:10.1530/ EJE-09-0322)

4 van der Lely AJ, Biller BM, Brue T, Buchfelder M, Ghigo E, Gomez R, Hey-Hadavi J, Lundgren F, Rajicic N, Strasburger CJ et al. Long-term safety of pegvisomant in patients with acromegaly: comprehensive review of 1288 subjects in ACROSTUDY. Journal of Clinical Endocrinology and Metabolism 201297 1589-1597. (doi:10.1210/jc.2011-2508)

5 Marazuela M, Lucas T, Alvarez-Escola C, Puig-Domingo M, de la Torre NG, de Miguel-Novoa P, Duran-Hervada A, Manzanares R, Luque-Ramirez M, Halperin I et al. Long-term treatment of acromegalic patients resistant to somatostatin analogues with the GH receptor antagonist pegvisomant: its efficacy in relation to gender and previous radiotherapy. European Journal of Endocrinology 2009160 535-542. (doi:10.1530/EJE-08-0705)

6 Parkinson C, Burman P, Messig M \& Trainer PJ. Gender, body weight, disease activity, and previous radiotherapy influence the response to pegvisomant. Journal of Clinical Endocrinology and Metabolism 200792 190-195. (doi:10.1210/jc.2006-1412) 
7 Neggers SJ, van Aken MO, Janssen JA, Feelders RA, de Herder WW \& van der Lely AJ. Long-term efficacy and safety of combined treatment of somatostatin analogs and pegvisomant in acromegaly. Journal of Clinical Endocrinology and Metabolism 200792 4598-4601. (doi:10.1210/jc.2007-1234)

8 Andreassen M, Frystyk J, Faber J \& Kristensen LO. GH activity and markers of inflammation: a crossover study in healthy volunteers treated with GH and a GH receptor antagonist. European Journal of Endocrinology 2012166 811-819. (doi:10.1530/ EJE-11-1009)

9 Junnila RK, Strasburger CJ \& Bidlingmaier M. Pitfalls of insulinlike growth factor-i and growth hormone assays. Endocrinology and
Metabolism Clinics of North America 201544 27-34. (doi:10.1016/ j.ecl.2014.10.003)

10 Droste M, Domberg J, Buchfelder M, Mann K, Schwanke A, Stalla G $\&$ Strasburger CJ. Therapy of acromegalic patients exacerbated by concomitant type 2 diabetes requires higher pegvisomant doses to normalise IGF1 levels. European Journal of Endocrinology 2014171 59-68. (doi:10.1530/EJE-13-0438)

11 Leung KC, Doyle N, Ballesteros M, Waters MJ \& Ho KK. Insulin regulation of human hepatic growth hormone receptors: divergent effects on biosynthesis and surface translocation. Journal of Clinical Endocrinology and Metabolism 200085 4712-4720. (doi:10.1210/ jc.85.12.4712)

Received 4 January 2016

Revised version received 28 June 2016

Accepted 7 July 2016 